\title{
L'insertion professionnelle des finissantes et des finissants du Conservatoire de musique du Québec
}

\section{DENIS PERRON \& RENÉE CLOUTIER}

Université Laval

\section{Résumé}

Bien que le Conservatoire de musique du Québec (CMQ) ait franchi le demi-siècle d'histoire, peu de renseignements concernant les origines et les devenirs de ses finissantes et ses finissants ont été produits jusqu'à ce jour. Ainsi, nous avons constitué une banque de données portant sur cette clientèle, à partir des promotions des années 1988 à 1991, et c'est à l'aide de ces données que nous avons cherché à comprendre ce qu'il advient des individus qui ont complété une formation au CMQ.

Afin de mieux saisir les facteurs d'influence de l'insertion professionnelle de cette clientèle - sujet au centre de cet article -, nous avons tenu compte du sexe, de l'origine familiale, de l'âge du début des apprentissages musicaux, de l'ordre d'enseignement complété, de la discipline principale étudiée ainsi que de la note décernée par l'Institution.

Les auteurs désirent remercier les finissantes et les finissants des différents conservatoires de musique du Québec qui ont pris de leur temps afin de répondre au questionnaire qui a donné lieu à la recherche à l'origine de cet article. Un grand merci également aux différents membres de la Direction générale du Conservatoire de musique du Québec qui ont apporté un soutien important, plus particulièrement à Pierre Thibault, ex-directeur général de l'Institution. 
Il serait intéressant de vérifier avec attention si une proportion importante de ces individus poursuivent leur formation musicale après le CMQ. Toutefois, nous savons que parmi celles et ceux qui occupent des emplois à la suite de ces études, plus de $80 \%$ le font dans des domaines correspondant à leur formation musicale.

\section{Abstract}

Although the Conservatoire de musique du Québec (CMQ) has existed for half a century, to date very little information has been compiled on the origins of its students or on how its graduates have fared. We have thus produced a data bank on this clientele, covering students who graduated from the CMQ between 1988 and 1991, and used it to determine how their carrers have progressed since this time.

In order to better comprehend the factors influencing the students' integration into the labour market, which is the aim of the article, we have taken into account gender, family background, age at which musical training began, levels of schooling completed, main area of study, and the grades obtained from the institution.

It would be interesting to determine whether a large proportion of these individuals pursued their musical training after graduating from the CMQ. We do know, however, that over $80 \%$ of employed CMQ graduates found jobs in a field related to their studies.

\section{Conservatoire de musique du Québec}

Le 29 mai 1942, l'assemblée législative du Québec votait la loi instituant le Conservatoire de musique et d'art dramatique (Duchesnay 1980, p. 1). ${ }^{1}$ Cette idée de créer une école musicale de plan européen et prise en charge entièrement par l'État était vieille de plus de soixante-sept ans déjà. Dans un esprit de démocratisation de l'enseignement déjà entreprise au Québec, le Conservatoire devait assurer la gratuité scolaire pour toute candidate ou tout candidat démontrant des aptitudes, à la suite d'auditions entendues en ce sens. Quoique les pionniers furent nombreux à préparer sa réalisation - Calixa Lavallée, Léo-Pol Morin, Claude Champagne, Achile Fortier (et d'autres) -, c'est à Wilfrid Pelletier qu'on 
doit la concrétisation de ce projet avec les collaborations de Jean Vallerand et de Claude Champagne.

Le premier conservatoire répondant à ces caractéristiques, et qui vit le jour au Québec, fut celui de Montréal. Aujourd'hui, le Conservatoire de musique du Québec (CMQ) dénombre sept composantes : Montréal (1942), Québec (1944), Trois-Rivières (1964), Val-D’Or (1965), Hull et Chicoutimi (1967) et Rimouski (1973). De plus, un demi-siècle d'histoire a été franchi depuis sa création et actuellement, l'ensemble des diplômés du quatrième cycle, toutes disciplines confondues, se situe audelà des mille individus (Painchaud 1992, pp. 77-81).

La formation dispensée au CMQ comporte quatre ordres d'enseignement qui sont identifiés par un échelon numérique de un à quatre. Toutefois, nous retrouvons aussi le pré-cycle qui offre aux plus jeunes une formation préparatoire. Les deux cycles qui nous intéressent dans cet article sont les troisième et quatrième cycles. Ils correspondent approximativement au baccalauréat et à la maîtrise universitaires, c'està-dire aux études supérieures.

L'objectif visé par l'Institution consistait dès ses débuts, et encore de nos jours, à former des musiciens professionnels pour le Québec. «Le Conservatoire de musique du Québec a pour but premier de former des musiciens professionnels par un enseignement spécialisé et intensif $[\ldots]$ » (Thibaut 1991, p. 9).

Le 3 mai 1994, l'Assemblée nationale du Québec adoptait la loi 135. Celle-ci vint modifier plusieurs aspects de l'ancienne loi sur le Conservatoire (L.R.Q., chapitre C-62). Dorénavant, la gratuité scolaire est remise en question ce qui, pour plusieurs, constitue une menace pour la démocratisation de l'enseignement. De plus, la direction de l'Institution sera confiée à un conseil d'administration autonome plutôt que de relever directement du ministère de la Culture du Québec. Notre analyse est à l'effet que l'État se décharge de ses responsabilités par rapport au Conservatoire de musique et d'art dramatique du Québec. De plus, la mission de certaines composantes du réseau est appelée à des modifications importantes. La loi 135 n'est cependant toujours pas en application actuellement, près de deux ans après sa sanction définitive (c'est-à-dire le 5 mai 1994). 


\section{Objectifs de l'étude et angle d'analyse}

L'objectif principal de cette étude porte sur l'insertion professionnelle des personnes diplômées du Conservatoire de musique du Québec. Nous avons voulu examiner dans quelle mesure ces finissantes et ces finissants travaillent dans leur champ de formation, et dans le secteur visé par l'institution de formation, soit celui des orchestres symphoniques. Nous avons cherché à savoir si ce groupe qui avait fréquenté le Conservatoire de musique du Québec possédait en outre une «carte d'identité» distinctive et comportant un dénominateur commun en rapport avec son milieu socioculturel et socio-économique d'origine.

Nous avons affaire ici à un groupement particulier selon la terminologie de Gurvitch. ${ }^{2}$ En effet, ces personnes font partie d'un groupe spécifique lié par un titre scolaire acquis et partagent aussi, de façon importante, un objectif commun : travailler comme musiciennes ou comme musiciens professionnels. Ainsi, les populations sont généralement organisées en sociétés ou en groupes particuliers, associés à des pairs par des affiliations, des alliances ou des idéaux qu'elles partagent. Cependant les résultats des analyses sociologiques nous font voir que les groupes se distinguent eux-mêmes en sous-populations. Dans quelle mesure les cheminements particuliers dans l'organisation scolaire (ordre d'enseignement, réussite scolaire, spécialisation) vont-ils distinguer ces diplômés et interagir sur leur insertion professionnelle? De façon plus importante, dans quelle mesure leurs diverses expériences familiales, notamment dans le champ culturel, vont-elles les marquer différemment et influer sur leurs cheminements académiques et sur leur insertion professionnelle?

Nous faisons ici référence à la notion de capital incorporé de Bourdieu lorsqu'il traite de la transmisssion culturelle au sein de la famille.

«[...] les différences dans le capital culturel possédé par la famille impliquent des différences d'abord dans la précocité du commencement de l'entreprise de transmission et d'accumulation, avec pour limite la pleine utilisation de la totalité du temps biologiquement disponible, le temps libre maximum étant mis au service du capital culturel maximum, ensuite 
dans la capacité ainsi définie de satisfaire aux exigences proprement culturelles d'une entreprise d'acquisition prolongée» (Bourdieu, 1979, pp. 4-5).

Cette dernière caractéristique, fondamentale dans la démarche de la recherche qui a inspiré cet article (Perron, 1995), est aussi à la base des variables retenues afin de tenter de répondre à la question initiale suivante: Qu'advient-il des individus qui ont complété une formation au Conservatoire de musique du Québec ?

La relation formation/emploi dont il sera question dans cet article renvoie aux emplois présentant un lien avec la formation dispensée au CMQ.

«La vie musicale peut se diviser en deux pôles: d'un côté, les activités musicales comme telles (interprétation et composition) et, de l'autre, les activités para-musicales, comme l'enseignement, l'administration artistique, le journalisme musical et bien d'autres» (Rousseau, 1986, p. 3).

De ce point de vue, la vie active professionnelle ou l'insertion professionnelle des musiciennes et des musiciens comporte ses particularités et nous examinerons cette réalité à partir de l'objectif poursuivi par l'Institution qui vise à former des musiciens professionnels essentiellement. Par conséquent, nous analyserons l'insertion professionnelle de ce groupe d'individus en rapport aux activités musicales seulement.

Contrairement à d'autres secteurs d'activités professionnnelles qui exigent une formation postsecondaire, celui des arts (notamment de la musique) n'est pas toujours prisé par les parents de classe sociale supérieure comme champ priviligié d'insertion professionnelle. Ceci augmente l'intérêt de l'analyse. Une étude française (Hennion et al., 1983) indique que les ambitions initiales des individus - de poursuivre des études en musique - varient selon les milieux sociaux. Pour ceux qui sont issus d'origines culturelle et économique défavorisées, ${ }^{3}$ le Conservatoire constituerait un facteur d'ascension sociale important, audelà des pratiques musicales existant dans la famille.

«Les ambitions initiales du sujet dépendent de son milieu social, $[\ldots]$ les visées professionnelles au départ sont plus 
fréquentes dans les milieux économiquement et culturellement défavorisés (29\%) que dans les milieux aisés $(18 \%) \gg($ p. 68).

D'autre part, d'autres facteurs peuvent intervenir sur l'insertion professionnelle des personnes diplômées des Conservatoires de musique; pensons par exemple aux facteurs reliés à l'offre de travail. Dans la société occidentale moderne, l'équilibre entre l'offre et la demande de travail pourrait être un facteur très important sinon tributaire de la capacité qu'ont les individus de s'insérer professionnellement dans leur domaine respectif. Nous sommes conscients, dans le contexte économique actuel, de la fragilité de l'offre de travail, en particulier de celle provenant des orchestres symphoniques. Dans la présente étude, nous n'avons cependant pas abordé directement cette question de l'offre et de la demande de travail dans le domaine de la musique professionnelle.

L'approche sociologique dans laquelle s'inscrit l'étude à la base de cet article (Perron, 1995) vise un secteur de formation professionnelle encore peu analysé au Québec. Le développement des connaissances des trajectoires scolaires et de l'insertion professionnelle de ces finissantes et finissants, sur lequel porte cette étude, constitue donc un pas dans cette direction. Notre recherche s'inscrit dans cette perspective et nous tenterons de vérifier les liens entre la formation et l'emploi, mais aussi d'autres dimensions comme la stabilité de l'emploi pour ces individus.

Pour tenter de mieux comprendre cette réalité, cinq variables indépendantes ont été retenues - outre la variable dépendante de l'insertion professionnelle sur laquelle nous reviendrons plus loin - ainsi que deux variables à la fois indépendante et contrôle (la discipline principale choisie au CMQ et la récompense [ou note] obtenue). Des variables internes à l'organisation scolaire (ordre d'enseignement, réussite scolaire, spécialisation) ou externes à la formation reçue (statut socioculturel ou socio-économique des parents, sexe, âge du début des apprentissages musicaux) peuvent avoir des répercussions sur l'insertion professionnelle. Ces variables ainsi que leurs relations potentielles sont exposées au schéma $\mathrm{n}^{\circ} 1$ et décrites au schéma $\mathrm{n}^{\circ} 2$. Comme on peut le constater, d'autres relations que celles impliquant directement la variable dépendante (insertion professionnelle) font aussi l'objet d'une analyse et 


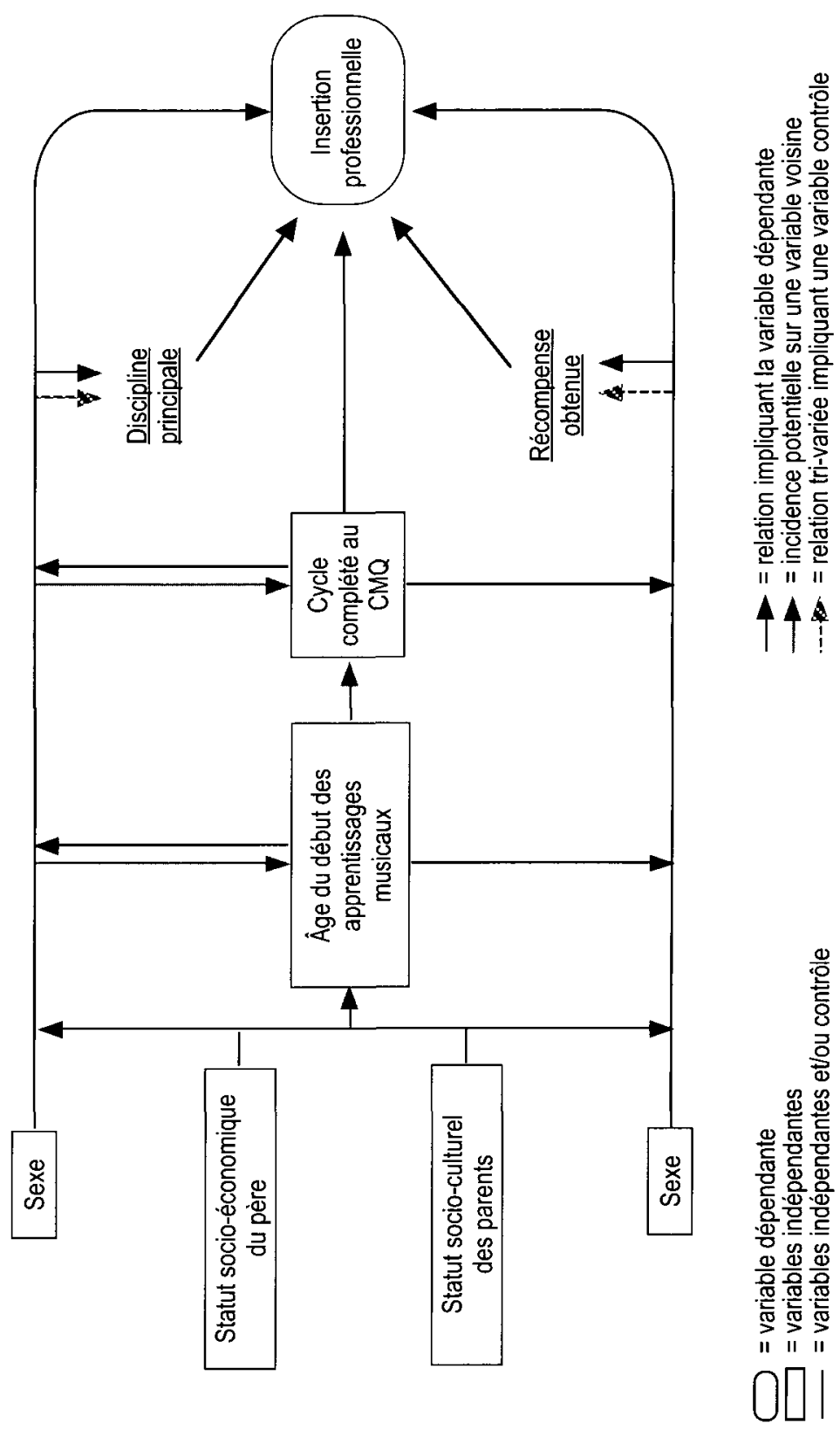


Variables identifiées dans l'étude

\begin{tabular}{|c|c|c|}
\hline Variables & & Indicateurs \\
\hline dépendante & - insertion professionnelle & $\begin{array}{l}\text { 1. correspondance formation/emploi ou non } \\
\text { 2. situation d'emploi (temps plein, partiel ou sans emploi) } \\
\text { 3. statut d'emploi (régulier ou temporaire) }\end{array}$ \\
\hline \multirow[t]{4}{*}{ indépendantes } & - sexe & 1. masculin ou féminin \\
\hline & $\begin{array}{l}\text { - statut socio-économique } \\
\text { du père }\end{array}$ & 1. statut élevé, moyen ou faible (selon l'emploi du père) \\
\hline & - statut socioculturel des parents & $\begin{array}{l}\text { 1. pratiques musicales dans la famille ou non } \\
\text { 2. activités socioculturelles des parents (élevées } \\
\text { moyennes ou faibles) }\end{array}$ \\
\hline & $\begin{array}{l}\text { - âge du début des apprentissages } \\
\text { musicaux des individus } \\
\text { - cycle complété au CMQ }\end{array}$ & $\begin{array}{l}\text { 1. tôt, âge moyen ou tardif } \\
\text { 1. troisième ou quatrième cycle }\end{array}$ \\
\hline indépendantes/contrôle & - discipline principale & $\begin{array}{l}\text { 1. instrumentale, non instrumentale ou plus d'une } \\
\text { 2. instrument "d'orchestre" ou autre instrument } \\
\text { 3. cordes frottées ou autre instrument }\end{array}$ \\
\hline
\end{tabular}

- récompense obtenue au CMQ 1. élevée ou faible 
dépendante (insertion professionnelle) font aussi l'objet d'une analyse et nous tenterons de vérifier si elles peuvent influencer le processus à l'étude.

\section{Population}

La population à l'étude est constituée d'anciens élèves ${ }^{4}$ qui ont complété un troisième et/ou un quatrième cycle au CMQ. Il s'agit plus précisément des promotions de finissantes et de finissants des années 1987-1988, 1988-1989, 1989-1990, 1990-1991, parmi l'ensemble des vingt-neuf disciplines principales offertes. ${ }^{5}$ Quoique seuls les individus qui ont complété un quatrième cycle - le cycle terminal - sont considérés comme finissants selon le CMQ, la population totale de l'étude inclut aussi les individus qui ont terminé un troisième cycle seulement, sans poursuivre par la suite, de façon à rendre compte de l'ordre d'enseignement dans le mécanisme d'insertion professionnelle. Cependant, cette caractéristique n'a pas semblé influencer de façon significative les variables à l'étude. De plus, nous parlons de population plutôt que d'échantillon puisque l'étude vise l'ensemble des finissantes et des finissants des promotions des années mentionnées un peu plus haut. La population est composée de 213 individus, provenant de l'une ou l'autre des sept composantes que comporte le CMQ (Chicoutimi, Hull, Montréal, Québec, Rimouski, Trois-Rivières et Val-D'Or). Ces composantes n'ont pas toutes la même importance en termes d'effectifs scolaires; en effet, plus de la moitié de la population totale $(57.3 \%)$ provient du Conservatoire de musique de Montréal, tandis que près du quart (23.0\%) provient de celui de Québec. Les cinq autres composantes du réseau québécois se partagent le reste de la population (graphique $\mathrm{n}^{\circ} 1$ ).

La population accessible, parmi les 213 finissantes et finissants regroupe 176 individus. De ce nombre, 124 personnes nous ont retourné leur questionnaire. ${ }^{6}$ Le taux de réponses correspond à $70.45 \%$. Soulignons que Relance Laval ${ }^{7}$ notait qu'en Arts, les diplômés étaient un peu plus difficiles à rejoindre que les autres populations de finissantes ou de finissants puisqu'ils résidaient souvent à l'extérieur du pays (Carrier et al., 1991, p. 11). 
Les graphiques $n^{\circ} 1, n^{\circ} 2$ et $n^{\circ} 3$ illustrent bien la constance des différentes répartitions de cette même population dans le processus d'enquête, soit la population totale d'origine, la population accessible et, en fin de compte, la population des répondantes et des répondants. L'équilibre ainsi maintenu ajoute une dimension aux données recueillies, surtout quant à leur aspect représentativité. Mentionnons en outre que la population des finissantes et des finissants des promotions à l'étude comprend trois fois plus d'individus issus du quatrième cycle que du troisième. Ceci démontre que le plus souvent, les finissantes et les finissants du troisième cycle poursuivent au quatrième cycle, à la suite de leur démarche (Perron, 1995, p. 76).

\section{Le concept d'insertion professionnelle}

Selon Tanguy (1986, pp. 49-50), «ll faut attendre le milieu des années 1960 pour voir divers organismes engager des études où l'on tente de suivre l'évolution au cours du temps d'une promotion de jeunes scolaires, jusqu'à son passage à la vie active». Le rapport à l'emploi et le marché du travail intéresse, depuis, bon nombre de gestionnaires ou de chefs d'établissements scolaires (entre autres) désirant connaître le devenir de leurs élèves, à la suite de leur formation.

Graphique $n^{\circ} 1$

Population totale par conservatoire (213 individus)

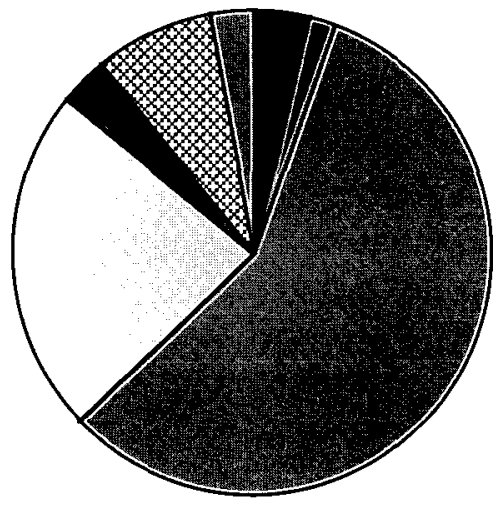

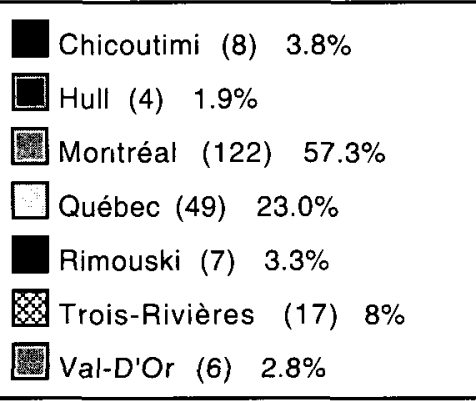




\section{Graphique $n^{\circ} 2$}

\section{Population accessible par conservatoire (176 individus)}

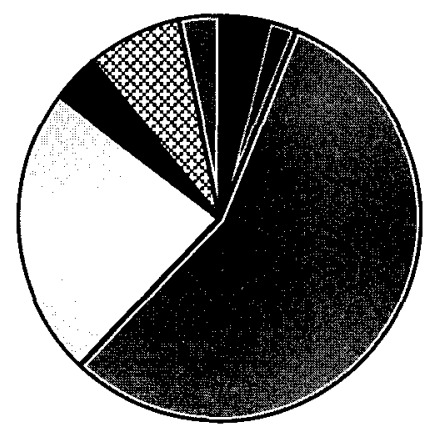

Chicoutimi (7) $4 \%$
$\square$ Hull (4) $2.3 \%$
$\square$ Montréal (98) $55.7 \%$
$\square$ Québec (41) $23.3 \%$
Rimouski (7) $4 \%$
Trois-Rivières (13) $7.4 \%$
Val-D'Or (6) $3.4 \%$

Graphique $n^{\circ} 3$

Population des répondants par conservatoire (123 individus)

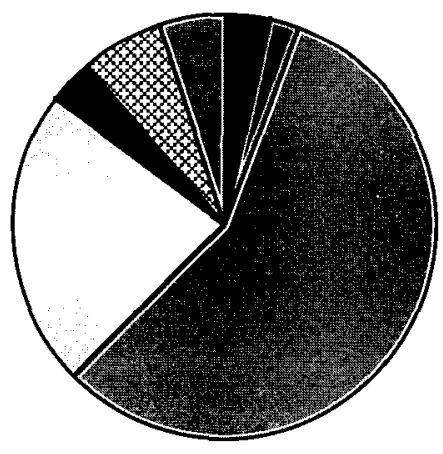

Chicoutimi (4) $3.3 \%$

Hull (3) $2.4 \%$

Montréal (70) $56.9 \%$

$\square$ Québec (28) 22.8\%

Rimouski (4) $3.3 \%$

Trois-Rivières (8) $6.5 \%$

圈 Val-D'Or (6) $4.9 \%$ 
Le concept d'insertion professionnelle n'est cependant pas facile à circonscrire puisqu'on constate qu'aucune définition ne satisfait totalement l'ensemble des chercheuses et des chercheurs qui s'intéressent à la question. Il existe pourtant des auteurs qui circonscrivent de façon fort valable le concept d'insertion professionnelle (Lamoure \& Viney, 1982; Tanguy, 1986; Vincens, 1989; etc.) mais leur approche ne correspond pas entièrement à la nature de notre étude puisque la formation de musicienne ou de musicien professionnel - telle que dispensée par le $\mathrm{CMQ}^{8}$ - ne laisse entrevoir que peu de débouchés autres que ceux reliés à la musique professionnelle. De plus, il faut préciser que dans le présent article, nous ne retiendrons que le concept d'insertion professionnelle, bien que l'étude qui a inspiré cet article traite également des aspirations professionnelles de ces finissantes et ces finissants.

Dans le cadre de cette étude, nous retiendrons deux idées maîtresses inspirées de la recension des écrits à cet égard - afin de définir le concept. Par contre, ces deux idées seront traitées de façon indépendantes l'une de l'autre; la dimension réduite de notre population invitant à la prudence.'

D'une part, afin de définir l'insertion professionnelle, nous retenons la relation formation/emploi qui distingue et lie ces individus par un commun dénominateur - la formation - comme nous l'avons souligné.

«Dans tous les travaux sur l'insertion, se retrouve l'hypothèse de la finalité professionnelle des études [...]. Une nécessité "technique" selon laquelle les connaissances et les savoirfaire, donnés par la formation, sont indispensables pour accomplir les tâches constitutives de l'emploi» (Vincens, 1989, pp. 5-6).

Nous retenons aussi la dimension de la stabilité de l'emploi, même si cette notion semble plutôt éphémère, ou rarement acquise, dans le domaine de la musique professionnelle. Essentiellement, Dupaquier (1986) indique que pour être inséré, un individu ne doit plus investir d'énergie à la recherche d'un emploi et que celui-ci doit être satisfait des conditions qui prévalent, là où il travaille. Précisons que cette dimension du concept de l'insertion professionnelle, qu'est la stabilité, sera mesurée à partir de deux indicateurs: la situation de l'emploi (temps plein, temps partiel ou sans emploi) et le statut de l'emploi (régulier ou temporaire) pendant la semaine contrôle. ${ }^{10}$ 


\section{Aspect méthodologique}

Afin de réaliser cette étude sur les finissantes et les finissants du CMQ, il a fallu constituer une banque de données. Malgré la faible dimension de la population en cause, nous avons opté pour une méthodologie quantitative de façon à présenter une vision d'ensemble de la situation caractérisant ces individus. Le mode d'administration retenu a été le questionnaire par la poste. En effet, cette technique semblait bien appropriée, principalement parce que la population visée était répartie sur un territoire relativement vaste. De plus, l'outil s'adressait à une population homogène, et cette caractéristique était elle aussi propice à ce mode d'administration. Quoique le principal désavantage connu de cette approche soit la sensibilité au phénomène de non-réponses, les résultats à cet égard ont été très satisfaisants, probablement grâce à la rigueur dans la tenue à jour des retours de questionnaires qui a permis de loger systématiquement des rappels auprès des retardataires.

Le questionnaire qui a servi à la cueillette des données comporte quelque 92 questions et reprend en grande partie celui qui a été utilisé et validé par Relance Laval (Carrier et al., 1991). De plus, certaines questions ont aussi été inspirées des travaux d'Archambault et al. (1990) ainsi que de Hennion et al. (1983) - tous deux de France.

En accord avec les résultats de Hennion et al. (1983) et de Perron (1995), un certain nombre de variables ont été retenues et analysées dans le cadre du présent article. Voir les schémas $n^{\circ} 1$ et $n^{\circ} 2$ qui exposent ces variables, ainsi que leurs composantes. Précisons enfin que les données de cette étude ont été traitées à l'aide du logiciel «Statistical Package for Sociological Science», version Xe (S.P.S.S.X.).

\section{Portrait de la population}

\section{Insertion professionnelle}

Dans cette partie, nous présentons les distributions de fréquences des variables retenues pour les fins de cet article dans le but de tracer un portrait global de la population à l'étude. Parmi les individus qui ont déclaré occuper des emplois pendant la semaine contrôle, $81.8 \%$ des 
Tableau $n^{\circ} 1$

\section{L'insertion professionnelle (3 indicateurs)}

$\mathrm{N} \quad \% \quad(\%)$

Correspondance formation/emploi

$\begin{array}{lrr}\text { oui } & 72 & 81.8 \\ \text { non } & 16 & 18.2 \\ \text { total } & 88 & 100.0\end{array}$

Situation de l'emploi

$\begin{array}{lrrc}\text { temps plein } & 45 & 37.8 & (48.9) \\ \text { temps partiel } & 47 & 39.5 & (51.1) \\ \text { sans travail } & 27 & 22.7 & (-) \\ \text { total } & 119 & 100.0 & (100.0)\end{array}$

Statut de l'emploi

$\begin{array}{lrr}\text { régulier } & 40 & 44.9 \\ \text { temporaire } & 49 & 55.1 \\ \text { total } & 89 & 100.0\end{array}$

personnes occupaient des emplois en relation avec leur formation tandis que $18.2 \%$ travaillaient dans des domaines sans relation avec leur formation (tableau $\mathrm{n}^{\circ} 1$ ).

D'autre part, pendant la même période, $22.7 \%$ des répondantes et des répondants étaient sans travail; $37.8 \%$ occupaient des emplois à temps plein, tandis que $39.5 \%$ occupaient des emplois à temps partiel. Toutefois, si on élimine de cette population les individus qui se retrouvaient sans travail, $48.9 \%$ des travailleuses et des travailleurs occupaient des emplois à temps plein, tandis que $51.1 \%$ occupaient des emplois à temps partiel.

Enfin, $44.9 \%$ des travailleuses des travailleurs occupaient des emplois réguliers pendant la semaine contrôle, tandis que $55.1 \%$ occupaient des emplois temporaires. Rappelons que la situation ainsi que le statut d'emploi sont des indicateurs qui, dans cette étude, servent à mesurer la 
stabilité des emplois. De plus, la stabilité est elle-même un indicateur de la variable dépendante relative à l'insertion professionnelle.

On constate qu'en dépit du fait qu'assez souvent, ces individus travaillent dans des conditions précaires (travail à temps partiel et/ou temporaire), les personnes qui travaillent occupent toutefois des emplois dans leur domaine pour la grande majorité (81.8\%). La stabilité de l'emploi est néanmoins rarement acquise pour cette catégorie de travailleuses et de travailleurs en quête de contrats dans leur domaine spécifique. Les musiciens québécois (en général) occupent fréquemment des emplois instables. En effet:

«[...] la quasi-totalité des membres de la Guilde des musiciens travaillent comme pigistes. [...] Pour vivre du travail à la pige, il faut que le musicien soit prêt à passer la moitié de son temps à courir après les contrats») (Rousseau, 1986, p. 5).

\section{Pratiques musicales familiales}

Dans un tout autre ordre d'idées, examinons l'existence de pratiques musicales dans la famille. Ces pratiques réfèrent à celles de tous les membres de la famille immédiate, pas seulement des parents. Lorsqu'il y a lieu, il s'agit donc de celles qui existaient avant même que la répondante ou le répondant commence sa première leçon musicale. Ainsi, on constate que les répartitions sont relativement uniformes puisque $51.6 \%$ disent que de telles pratiques musicales existaient dans leur famille avant même de commencer des leçons musicales, contre $48.4 \%$ qui indiquent que de telles pratiques familiales n'existaient pas. Cette question nous intéresse particulièrement puisque nous pensons que la fréquentation du Conservatoire référerait souvent à la reproduction d'un comportement existant au sein de la famille. En effet;

«plus que le goût pour la musique (des parents ou de l'enfant), la pratique instrumentale aiguille de façon décisive l'enfant vers l'école de musique: c'est elle que les parents "pratiquants" tiennent à transmettre à leur enfant, elle que l'enfant cherche à imiter lorsque cette pratique existe dans la famille» (Hennion et al., 1983, p. 44). 
Tout comme à la façon dont l'origine sociale affecte le cursus des étudiants dans leurs fréquentations postsecondaires en général, ${ }^{11}$ l'influence de l'origine socioculturelle reliée à de telles pratiques familiales pourrait être déterminante quant à la poursuite d'études musicales et peut-être quant à l'insertion professionnelle par la suite.

\section{Activités socioculturelles des parents}

De même, les activités socioculturelles des parents (en général) nous intéressent aussi. C'est à partir des réponses obtenues à six questions portant sur divers types d'activités socioculturelles des parents que nous avons tenté de tracer un portrait du contexte familial des répondantes et des répondants à cet égard. Essentiellement, les individus étaient invités à répondre à ces questions selon une échelle hiérarchique.

Nous voulions ainsi savoir de quels milieux, en rapport au niveau d'activités socioculturelles des parents, provenaient les finissantes et les finissants du CMQ, mais aussi si ce niveau d'activités socioculturelles des parents pouvait influencer, par la suite, l'insertion professionnelle des individus qui ont complété une formation au CMQ. En d'autres termes, la démarche qui consiste à entreprendre des études musicales jusqu'à ce niveau de formation peut-elle être la conséquence de la transmission d'un capital culturel des parents? Nous ne pouvons répondre de façon exhaustive à cette question, mais nous avons tenté de décrire le profil de la population, quant à diverses caractéristiques sociologiques, afin de mieux comprendre l'influence potentielle de ces origines sur le curriculum.

Pour ce qui est de la variable propre aux activités socioculturelles des parents, nous avons distribué la population en trois catégories selon la courbe normale. Cette variable a été constituée à partir de six paramètres ${ }^{12}$ que nous avons distribués en trois catégories par la suite. En pareil cas, il est d'usage de tendre vers les proportions les plus près possibles de $15 \%, 70 \%$ et $15 \%$. Ainsi, $15.9 \%$ des répondantes et des répondants sont issus de familles où les parents avaient des activités socioculturelles considérées comme élevées. La majorité des répondantes et des répondants $(70.8 \%)$ sont issus de familles où les parents avaient des activités socioculturelles moyennes, tandis que 
Tableau ${ }^{\circ} 2$

\section{Statut socioculturel des parents ( 2 indicateurs)}

Pratiques musicales dans la famille

$\begin{array}{lrr}\text { oui } & 63 & 51.6 \\ \text { non } & 59 & 48.4 \\ \text { total } & 122 & 100.0\end{array}$

Activités socioculturelles des parents

$\begin{array}{lrr}\text { élevées } & 18 & 15.9 \\ \text { moyennes } & 80 & 70.8 \\ \text { faibles } & 15 & 13.3 \\ \text { total } & 113 & 100.0\end{array}$

Statut socio-économique du père (1 indicateur)

Statut socio-économique du père

$\begin{array}{lrr}\text { faible } & 18 & 15.3 \\ \text { moyen } & 86 & 72.9 \\ \text { élevé } & 14 & 11.9 \\ \text { total } & 118 & 100.0\end{array}$

$13.3 \%$ proviennent de familles où les parents avaient des activités socioculturelles faibles (tableau $n^{\circ} 2$ ).

Si les pratiques musicales existant au sein de la famille semblent plus élevées que l'ensemble de la population, pour les finissantes et les finissants du CMQ (51.6\%), nous ne pouvons nous prononcer quant aux activités socioculturelles des parents étant donné la distribution constituée selon la courbe normale, comme mentionné plus haut.

\section{Statut socio-économique du père}

Pour évaluer le statut socio-économique du père, nous avons eu recours à l'échelle de Blishen et al. (1987). Les diverses réponses obtenues à la question: "Quelle a été l'occupation principale de votre père durant la 
majeure partie de sa vie ?" ont été codées selon cette échelle. Cette classification de Blishen et al. (1987) tient compte du prestige social référant à la fois au revenu et au niveau de scolarité ou d'éducation de l'occupation. Dans le cas qui nous intéresse, il ressort trois catégories distinctes que nous avons volontairement distribuées selon une courbe normale. En soi, cette distribution nous renseigne uniquement sur l'origine socio-économique des individus entre eux, mais non sur cette origine de façon absolue. Toutefois, cette distribution prend tout son sens lorsqu'on tente des croisements bi-variés ou même tri-variés avec un des indicateurs de la variable dépendante, l'insertion professionnelle, et une variable contrôle, le cas échéant.

Selon la codification de Blishen et al. (1987), voici à quels types d'emplois réfèrent les points de coupe supérieurs des trois catégories. Le statut socio-économique faible réfère à la catégorie gardiens et préposés à des services de sécurité (code 31.95 ); le statut socio-économique moyen à celle de professeurs au niveau secondaire (code 70.19) et finalement le statut socio-économique élevé à la catégorie médecins et chirurgiens (code 99.99). ${ }^{13}$

Il est pratiquement impossible, même en distribuant la population selon la courbe normale, d'obtenir des regroupements parfaitement équilibrés $(15 \%, 70 \%$ et $15 \%)$. Ainsi, selon l'emploi du père, $72.9 \%$ des répondantes et des répondants se retrouvent dans la catégorie statut socio-économique moyen, $15.3 \%$ dans la catégorie statut socioéconomique faible et $11.9 \%$ dans la catégorie statut socio-économique élevé (tableau $\mathrm{n}^{\circ} 2$ ).

\section{Âge, cycle complété et sexe}

Trois autres variables indépendantes ont aussi été retenues dans cette étude: l'âge du début des apprentissages musicaux, le cycle complété au CMQ et le sexe des répondantes et des répondants.

L'âge du début des apprentissages musicaux a été distribué en trois catégories (tableau $\mathrm{n}^{\circ} 3$ ), inspirées de celles évoquées par Hennion et al. (1983, p. 68) dans leur étude. Ainsi, on retrouve $31.1 \%$ des répondantes et des répondants ayant débuté leurs pratiques musicales tôt ( 7 ans et moins), $17.2 \%$ à un âge moyen ( $8-9$ ans) et $51.6 \%$, tardivement (10 ans 
Tableau ${ }^{\circ} 3$

Âge du début des apprentissages musicaux (1 indicateur)

Âge du début des apprentissages

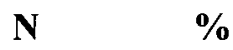

musicaux des individus

$\begin{array}{lrr}\text { tôt } & 38 & 31.1 \\ \text { moyen } & 21 & 17.2 \\ \text { tardif } & 63 & 51.6 \\ \text { total } & 122 & 100.0\end{array}$

\section{Discipline principale (3 indicateurs)}

Discipline principale $\left(\mathrm{n}^{\circ} 1\right)$

instrumentale

non instrumentale

12

83.6

+ d'une discipline principale

8

9.8

total

122

6.6

100.0

Discipline principale $\left(\mathrm{n}^{\circ} 2\right)$

$\begin{array}{lrr}\text { associée à l'orchestre } & 68 & 66.7 \\ \text { non associée à l'orchestre } & 34 & 33.3 \\ \text { total } & 102 & 100.0\end{array}$

Discipline principale $\left(\mathrm{n}^{\circ} 3\right)$

cordes frottées

autres instruments

et plus). La population de cette étude tend donc à apporter un démenti quant à l'importance d'entreprendre des études à un jeune âge afin d'atteindre un niveau supérieur en musique. En effet, plus de la moitié des finissantes et des finissants du CMQ ont entrepris leur apprentissage musical tardivement, c'est-à-dire à partir de l'âge de 10 ans et plus (tableau $n^{\circ} 3$ ). 
Quant au cycle complété au CMQ, on remarque que les trois quarts des répondantes et des répondants sont des finissantes et des finissants $\mathrm{du}$ quatrième cycle $(76.4 \%)$, tandis que les autres ont complété un troisième cycle sans s'inscrire de nouveau au CMQ par la suite (23.6\%). La majorité des finissantes et des finissants ont rendu à terme (c'est-àdire jusqu'au cycle terminal) la formation qu'ils ont souvent entreprise depuis plusieurs années. Toutefois, il n'existe pas de relation statistiquement significative quant à la possibilité d'insertion professionnelle selon le cycle accompli au CMQ (troisième ou quatrième) par les individus.

Enfin, parmi les répondantes et les répondants, il y a autant de femmes que d'hommes qui ont terminé une formation au CMQ au cours des années visées par ces promotions. Cependant, il en est tout autrement pour certaines catégories de disciplines principales qui intéressent inégalement femmes et hommes. Nous pensons, entre autres, aux disciplines principales non instrumentales où les hommes se retrouvent presque exclusivement.

La variable indépendante/contrôle discipline principale complétée au CMQ a été mesurée à partir de trois indicateurs différents, ce qui implique autant de catégories réalisées. Le premier indicateur distingue donc les disciplines principales instrumentales, les disciplines principales non instrumentales et le regroupement de plus d'une discipline principale (instrumentale ou non). Le deuxième indicateur regroupe essentiellement les disciplines instrumentales et distingue les disciplines principales associées aux instruments traditionnels d'orchestre de type symphonique des disciplines instrumentales non traditionnellement associés à l'orchestre. Enfin, le troisième indicateur (essentiellement instrumental aussi) distingue les cordes frottées des autres disciplines principales instrumentales.

Par cette façon de distinguer, nous voulions mieux comprendre les différentes incidences ou relations entre variables, attribuables à un aspect particulier de la discipline principale choisie par l'individu. Par exemple, comme la grande majorité des individus sont inscrits en discipline principale instrumentale $(83.6 \%)$, nous voulions isoler cette clientèle et ainsi en constituer une catégorie à part. Parmi les disciplines 
principales instrumentales, celles-qui appartiennent aux cordes frottées revêtent une importance toute particulière car ces instrumentistes constituent majoritairement l'effectif des grands orchestres de type symphonique. Enfin, les instrumentistes associés à l'orchestre de type symphonique s'inscrivent dans la lignée des visées d'insertion professionnelle de l'Institution et l'orchestre constitue un des débouchés importants correspondant aux aspirations des élèves du CMQ. La distribution de fréquences selon les trois indicateurs de la variable discipline principale est présentée au tableau $n^{\circ} 3$.

$\mathrm{Au}$ quatrième cycle du CMQ, la récompense s'ajoute à la note lorsque cette dernière est égale ou supérieure à $75 \%$. Ainsi, on décerne un Premier prix aux individus qui obtiennent une note égale ou supérieure à $90 \%,{ }^{14}$ un Second prix aux individus qui obtiennent une note de $80 \%$ à $89 \%$, une Mention aux individus qui obtiennent une note de $75 \%$ à $79 \%$, tandis que ceux qui obtiennent une note de moins de $75 \%$ ne reçoivent pas de récompense. Comme instrument de mesure, la récompense représente beaucoup d'intérêt puisqu'elle constitue une classification réaliste à laquelle nous pouvons référer afin de distribuer la population. Bien que, pour l'Institution, la récompense n'existe pas au troisième cycle, nous avons utilisé cette même distribution avec cette population - à partir de la note qu'ils ont obtenue - afin d'établir une comparaison avec les individus qui ont complété un quatrième cycle.

Des quatre classifications que les récompenses impliquent, nous avons distribué la population de façon dichotomique en regroupant d'abord les individus qui ont obtenu les deux groupes de notes les plus élevées dans la première catégorie et tous les autres dans la seconde catégorie. Ainsi, les individus qui ont obtenu une note égale ou supérieure à $80 \%$ constituent la première catégorie (récompense ou note élevée) et tous les autres la seconde catégorie (récompense ou note faible). $\grave{A}$ partir de ces nouveaux regroupements, on constate que les récompenses obtenues sont souvent favorables aux finissantes et aux finissants puisque les trois quarts de la population ont ainsi obtenu une récompense (ou note) élevée (74.8\%), tandis que $25.2 \%$ des individus seulement ont obtenu une récompense (ou note) faible. 


\section{Analyse des résultats et interprétation des relations entre les variables}

L'objectif de cette étude est la compréhension du phénomène de l'insertion professionnelle des finissantes et des finissants du CMQ, à partir de la description des facteurs d'influence présumés que nous avons retenus. La démarche d'analyse de ces résultats comporte trois étapes. Comme l'indiquent les flèches du schéma $\mathrm{n}^{\circ} 1$, nous avons d'abord tenté de vérifier l'existence de relations bi-variées impliquant la variable dépendante (l'insertion professionnelle), ensuite nous avons tenté de vérifier l'incidence potentielle de certaines variables sur leurs variables voisines et enfin nous avons tenté de vérifier l'existence de relations trivariées en impliquant les variables contrôles. De plus, chaque relation à l'étude devait répondre à deux exigences pour qu'elle soit reconnue significative statistiquement. Premièrement, présenter un degré de signification (p) de point zéro cinq $(0.05)$ et moins et deuxièmement, présenter un coefficient de corrélation (l'intensité de la relation), mesuré selon le $\mathrm{V}$ de Cramer - pour lequel nous avons accepté une intensité égale ou supérieure à point deux (0.2). Les croisements de variables que nous avons tentés et qui n'ont pas satisfaits à ces exigences ont été considérés comme non significatifs statistiquement.

\section{1) Relations bi-variées avec la variable dépendante (l'insertion professionnelle) $^{15}$}

Chacune des sept variables apparaissant dans le schéma $\mathrm{n}^{\circ} 1$ et dans le schéma $n^{\circ} 2$ - traitée dans un premier temps comme une variable indépendante - a d'abord été analysée à partir de la relation potentielle avec la variable dépendante (insertion professionnelle).

Trois croisements ainsi obtenus présentent une corrélation significative statistiquement. La première corrélation concerne les activités socioculturelles des parents et la situation d'emploi des individus pendant la semaine contrôle (tableau $n^{\circ} 4$ ). On remarque d'abord que la majorité des individus qui étaient sans travail (52.9\%) proviennent de milieux où les activités socioculturelles des parents étaient élevées. Bien que nous ne puissions l'affirmer hors de tout doute, 
Tableau $\mathrm{n}^{\circ} 4$

Activités socioculturelles des parents et la situation d'emploi pendant la semaine contrôle (\%)

Activités socioculturelles des parents Situation d'emploi

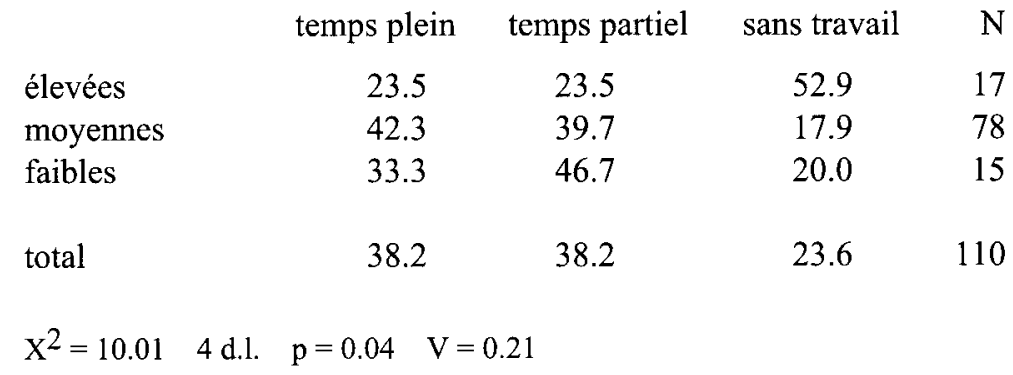

Instruments musicaux et la situation d'emploi pendant la semaine contrôle (\%)

Disciplines principales $\left(\mathrm{n}^{\circ} 2\right)$

Situation d'emploi

temps plein temps partiel sans travail N

\begin{tabular}{|c|c|c|c|}
\hline instruments d'orchestre & 31.3 & 38.8 & 29.9 \\
\hline autres disc. instrumentales & 50.0 & 41.2 & 8.8 \\
\hline total & 37.6 & 39.6 & 22.8 \\
\hline$x^{2}=6.49$ & & & \\
\hline
\end{tabular}

Disciplines principales $\left(\mathrm{n}^{\circ} 3\right)$

Situation d'emploi

temps plein temps partiel sans travail $\mathrm{N}$

cordes frottées

39.3

21.4

39.3

28

autres disc. instrumentales 37.0

46.6

16.4

73

total

37.6

39.6

22.8

101

$\mathrm{X}^{2}=6.49 \quad 2$ d.1. $\quad \mathrm{p}=0.03 \quad \mathrm{~V}=0.25$ 
la principale raison qui pourrait expliquer cette situation serait reliée au fait qu'une partie importante de cette clientèle poursuivrait des études et n'était donc pas disponible pour travailler à ce moment. Les deux autres groupes qui étaient sans travail pendant cette période se partagent de façon équilibrée puisque $17.9 \%$ sont des individus provenant de milieux où il $\mathrm{y}$ avait des activités socioculturelles des parents moyennes et que $20.0 \%$ proviennent de milieux où il y avait des activités socioculturelles faibles.

Parmi les individus qui travaillaient à temps plein pendant cette période, la plus faible proportion (23.5\%) provient de milieux où les activités socioculturelles des parents étaient élevées. Ce phénomène semble corroborer notre prétention à l'effet que ce groupe d'individus pourrait poursuivre des études après son passage au CMQ, et ainsi ne serait pas disponible pour du travail à temps plein. Selon la recension des écrits, on associe fréquemment milieux socioculturels et milieux socio-économiques élevés ou milieux socioculturels et milieux socioéconomiques défavorisés. Selon Hennion et al. (1983, p. 70), ces derniers sont marqués par un volontarisme plus fort et la seule venue au Conservatoire constitue une pratique sélective qui sort l'individu de son milieu. Ainsi, à la suite de leur formation complétée au CMQ, ils pourraient chercher davantage à s'insérer sur le marché du travail plutôt que de poursuivre des études additionnelles, en perfectionnement ou dans un tout autre domaine.

Les deuxième et troisième corrélations qui présentent une relation significative statistiquement sont toutes deux reliées à la discipline principale (catégories $n^{\circ} 2$ et $n^{\circ} 3$ ) et à la situation d'emploi pendant la semaine contrôle (tableau $n^{\circ} 4$ ). D'abord les instrumentistes associés à l'orchestre de type symphonique occupent des emplois à temps plein dans une proportion inférieure $(31.3 \%)$ à leurs collègues en instruments non associés à l'orchestre $(50.0 \%)$. Nous ne savons pas quelle est exactement la relation entre ces emplois et la formation en musique reçue au $\mathrm{CMQ}$. Toutefois, comme ces emplois sont occupés à temps plein, nous pouvons penser qu'il existe une relation avec la formation (directe ou indirecte) le plus souvent. Ainsi, les instrumentistes qui ne sont pas associés à l'orchestre de type symphonique occupent des emplois à temps plein dans une plus forte proportion que les autres. Ce 
champ de pratique ne rejoint pourtant pas les visées de l'Institution. Cette situation tend à indiquer que des débouchés potentiels - dans des domaines de la musique professionnelle - existent, bien qu'ils ne soient pas reliés spécifiquement aux visées de l'Institution. Les commentaires recueillis à la fin des questionnaires portent à penser qu'il est possible qu'il puisse s'agir, le plus souvent, de contrats professionnels reliés à la musique populaire ou commerciale.

Si nous comparons maintenant les instrumentistes aux cordes frottées $(39.3 \%)$ avec les autres (37.0\%), nous constatons qu'il n'y a pas de différence majeure au chapitre des emplois à temps plein, à partir de ce regroupement. Rappelons que les cordes frottées sont non seulement associées à l'orchestre, mais qu'elles constituent la très grande majorité de son effectif. De plus, cette famille instrumentale (cordes frottées) est tout à fait dans la visée de l'Institution, bien que ces instrumentistes se retrouvent sans travail - pendant la semaine contrôle - dans une proportion de $39.3 \%$, contre $16.4 \%$ pour les autres instrumentistes. Cette situation pourrait corroborer encore une fois notre prétention à l'effet qu'une proportion importante des clientèles types de l'Institution poursuivrait des études à la sortie du CMQ. Le quatrième cycle (cycle terminal) au CMQ correspond à la maîtrise dans l'ordre d'enseignement universitaire québécois. La concurrence des débouchés (les orchestres professionnels par exemple) pourrait faire en sorte qu'un perfectionnement devienne nécessaire à celles et ceux qui entrevoient de s'insérer professionnellement dans ce domaine. Ceci expliquerait - en partie du moins - pourquoi les individus dont la trajectoire correspond aux visées de l'Institution se retrouvent sans emploi dans des proportions supérieures aux autres, à la suite de la formation complétée au CMQ. Les données dont nous disposons actuellement ne nous permettent pas de vérifier spécifiquement ces allégations.

\section{2) Incidence de certaines variables sur leurs variables voisines ${ }^{16}$}

Parmi les variables voisines apparaissant au schéma $\mathrm{n}^{\circ} 1$, deux croisements présentent une relation significative statistiquement. Il s'agit d'abord du sexe et de la discipline principale (associé traditionnellement 
Tableau $n^{\circ} 5$

Discipline principale choisie selon le sexe (\%)

Sexe

Discipline(s) principale(s) choisie(s) instr. non instr. + d'une disc. pr. $\mathrm{N}$

$\begin{array}{lcccc}\text { féminin } & 93.4 & 0.0 & 6.6 & 61 \\ \text { masculin } & 73.8 & 19.7 & 6.6 & 61 \\ \text { total } & 83.6 & 9.8 & 6.6 & 122 \\ & & & & \\ \mathrm{X}^{2}=13.41 & 2 \text { d.1. } \quad \mathrm{p}=0.0 \quad \mathrm{~V}=0.33 & & \end{array}$

Activités socioculturelles des parents et âge du début des apprentissages musicaux (\%)

Activités socioculturelles des parents

Âge du début des appr. musicaux

$\begin{array}{lrccc} & \text { tôt } & \text { moyen } & \text { tardif } & \mathrm{N} \\ \text { élevées } & 55.6 & 11.1 & 33.3 & 18 \\ \text { moyennes } & 31.3 & 21.3 & 47.5 & 80 \\ \text { faibles } & 6.7 & 6.7 & 86.7 & 15 \\ \text { total } & 31.9 & 17.7 & 50.4 & 113 \\ \mathrm{X}^{2}=13.41 & 2 \text { d.1. } & \mathrm{p}=0.0 \quad \mathrm{~V}=0.33 & & \end{array}$

à l'orchestre de type symphonique ou non, catégorie $\mathrm{n}^{\circ} 2$ ), ainsi que des activités socioculturelles des parents et de l'âge du début des apprentissages musicaux (tableau $\mathrm{n}^{\circ} 5$ ).

Hennion et al. (1983, pp. 71-81) indiquent que pour les Français, les visées professionnelles et le choix d'un instrument de musique sont tous deux fortement sexués. Les filles sont plus nombreuses à fréquenter le Conservatoire de musique et s'y inscrivent plus tôt, elles proviennent de milieux plus aisés que les garçons et la pratique de la musique apparaît comme un élément nécessaire à la culture des jeunes filles de bonne 
famille beaucoup plus qu'à un choix de carrière, si on élimine l'enseignement. ${ }^{17}$

Parmi les finissantes et les finissants du CMQ, on ne dénote aucune femme ayant complété uniquement une formation dans une discipline principale non instrumentale ${ }^{18}$ contre $19.7 \%$ d'hommes. C'est donc dire que les disciplines principales instrumentales intéressent les femmes de façon très massive $(93.4 \%)$. Cette situation pourrait renforcer l'image stéréotypée de la pratique essentiellement instrumentale et caractéristique des élèves des conservatoires français que nous présente Hennion:

«apprendre à jouer du piano ou du violon n'est pas un investissement isolé, un choix indépendant, c'est l'un des éléments d'une identité sociale. En l'occurrence, la première image qui se dessine ici semble calquée sur un stéréotype classique de la musique classique - la jeune fille de bonne famille qui joue Chopin sur le piano du salon (Hennion et al. 1983, p. 41)».

Il est à remarquer qu'autant de femmes que d'hommes $(6.6 \%)$ ont cependant suivi plus d'une discipline principale, instrumentale ou non.

D'autre part, il existe une relation entre les activités socioculturelles des parents et l'âge du début des apprentissages musicaux des individus. Lorsque ces activités étaient élevées, les individus ont commencé tôt leur apprentissage musical (55.6\%). Inversement, lorsque les activités socioculturelles des parents étaient faibles, les individus ont commencé tardivement leur apprentissage musical $(86.7 \%)$. Ce croisement présente donc une relation négative puisque l'influence du milieu socioculturel familial est à l'inverse de la progression de l'âge où débutent les apprentissages musicaux des finissantes et des finissants du CMQ. En d'autres termes, plus les activités socioculturelles des parents étaient élevées, plus les apprentissages musicaux des individus ont débuté tôt et moins ces activités socioculturelles étaient élevées, plus les apprentissages musicaux des individus ont débuté tard. 


\section{3) Relations tri-variées impliquant des variables contrôles ${ }^{19}$}

Parmi 1'ensemble des relations évoquées au schéma $n^{\circ} 1$, trois croisements tri-variés présentent une corrélation significative statistiquement. D'abord, le statut d'emploi et la correspondance formation-emploi pendant la semaine contrôle selon le début des apprentissages musicaux (tôt). On constate que, parmi les individus qui ont entrepris leur apprentissage musical tôt et qui travaillaient de façon régulière pendant la semaine contrôle, la moitié (50.0\%) occupait des emplois correspondant à leur formation (tableau $n^{\circ} 6$ ). Par contre, parmi les individus qui occupaient des emplois temporaires, une majorité travaillait dans un domaine relié à leur formation (91.7\%). Ainsi, la correspondance formation-emploi, plus que le statut de l'emploi, est affectée positivement par le fait d'entreprendre des apprentissages musicaux tôt. Si on se réfère à la relation entre variables voisines, qui impliquait les activités socioculturelles des parents et l'âge du début des apprentissages musicaux, on peut dire que les origines socioculturelles de ces individus pourraient être déterminantes dans le processus d'insertion professionnelle, plus particulièrement quant à la correspondance entre l'emploi et la formation musicale. Nous rappelons que, dans cette étude, la correspondance formation-emploi est un des indicateurs retenu pour définir l'insertion professionnelle.

Le croisement tenté entre le statut d'emploi et la correspondance formation-emploi pendant la semaine contrôle selon la faible récompense (ou note) obtenue au CMQ est aussi significatif (tableau $\mathrm{n}^{\circ} 6$ ). Cette corrélation nous apprend que, malgré la faible note obtenue par ces individus à la suite de leur formation complétée au CMQ, ceux qui occupaient des emplois (réguliers et temporaires) pendant la semaine contrôle travaillaient dans des domaines correspondant à leur formation dans un large proportion (90.9\%). Plus précisément, parmi celles et ceux qui travaillaient de façon régulière, $75 \%$ occupaient des emplois correspondant à leur formation, tandis que tous les individus qui travaillaient de façon temporaire $(100.0 \%)$ occupaient des emplois correspondant à leur formation. Si pour ces individus, la poursuite d'études est possiblement moins attrayante en raison d'un moins grand 
Tableau $n^{\circ} 6$

Statut de l'emploi et correspondance formation-emploi pendant la semaine contrôle selon le début des apprentissages musicaux tôt (\%)

Statut d'emploi

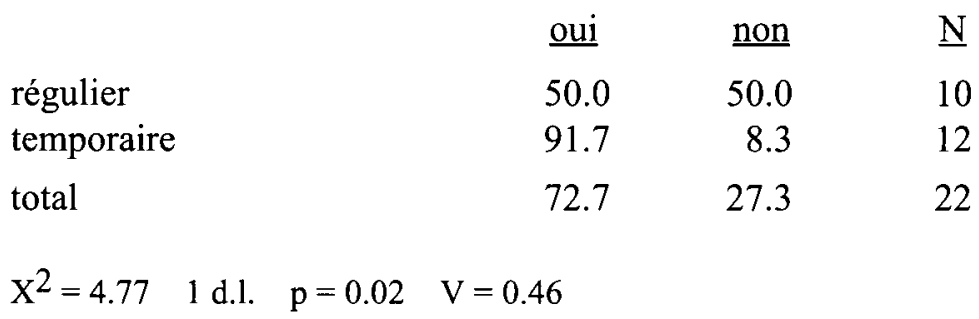

Statut d'emploi et correspondance formation-emploi pendant la semaine contrôle selon la récompense obtenue (faible) $(\%)$

Statut d'emploi

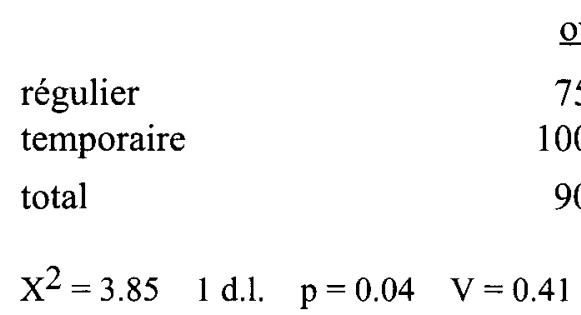

Correspondance formation-emploi $\underline{\text { oui }} \quad \underline{\text { non }} \quad \underline{\mathrm{N}}$

$\begin{array}{lll}75.0 & 25.0 & 8\end{array}$

$\begin{array}{lll}00.0 & 0.0 & 14\end{array}$

$90.9 \quad 9.1 \quad 22$

Statut socio-économique du père et correspondance formation-emploi pendant la semaine contrôle selon la pratique d'un instrument musical non traditionnellement associé à l'orchestre de type symphonique (\%)

Statut socio-économique du père Correspondance formation-emploi

\begin{tabular}{|c|c|c|c|c|}
\hline & & oui & non & $\underline{N}$ \\
\hline faible & & 100.0 & 0.0 & 5 \\
\hline moyen & & 82.6 & 17.4 & 23 \\
\hline élevé & & 0.0 & 100.0 & 2 \\
\hline total & & 80.0 & 20.0 & 30 \\
\hline$X 2=3.85$ & 1 d.l. $\quad p=0.04$ & $\mathrm{~V}=0.41$ & & \\
\hline
\end{tabular}


succès académique, nous pouvons penser que la récompense (ou note) obtenue au CMQ influencerait beaucoup plus les finissantes et les finissants ${ }^{20}$ que les employeurs susceptibles d'engager ces individus dans leur domaine. En effet, parmi les individus qui travaillaient pendant la semaine contrôle, ceux qui ont été les moins performants au CMQ occupaient tout de même de façon massive des emplois correspondant à leur formation.

Enfin, le statut socio-économique du père et la correspondance formation-emploi selon la pratique d'un instrument non traditionnellement associé à l'orchestre de type symphonique présente aussi une corrélation (tableau $\mathrm{n}^{\circ} 6$ ). Parmi ces instrumentistes, on constate que tous les individus (100.0\%) qui travaillaient pendant la semaine contrôle et dont le statut socio-économique du père est faible occupaient des emplois correspondant à leur formation. À l'inverse, aucun des individus qui travaillaient pendant la même période et dont le statut socio-économique du père est élevé n'occupait un emploi correspondant à sa formation. Cette observation semble valider - aussi bien pour le Québec - la thèse que présentait Hennion et al. (1983) dans leur étude à l'effet que, pour les individus d'origines populaires, les emplois dans le domaine de la musique professionnelle constituent une ascension sociale et sont marqués par un volontarisme plus fort. Il en serait donc autrement pour les individus dont le statut socio-économique du père est élevé, puisque ces personnes considéreraient la musique différemment; ainsi, la volonté d'en faire profession ne représenterait pas une ascension sociale. De plus, les instruments non associés à l'orchestre de type symphonique ne rejoignent pas les visées d'insertion professionnelle du CMQ et sont le plus souvent associés à la musique populaire. Il est possible que ces instruments caractérisent l'origine des individus comme il semble que ce soit le cas en France.

«Les instruments sont très inégalement choisis selon les milieux sociaux et leurs pratiques musicales [...] les vents, en particulier les cuivres, sont avant tout des instruments populaires (33\% chez les ouvriers, $21 \%$ dans les classes moyennes, 8\% dans les classes aisées) (Hennion et al. 1983, pp.79-80)». 


\section{Conclusion}

Parmi les différents croisements qui ont été tentés dans cette étude, certains n'ont pas démontré de relation significative statistiquement. Ceci ne signifie pas nécessairement qu'il n'y ait aucune relation entre ces variables. Cependant, cette situation nous invitant à la prudence, nous avons préféré nous abstenir de tirer des conclusions à leur effet.

Outre la relation formation-emploi et la stabilité qui définissent la variable dépendante (l'insertion professionnelle), l'identité et les cheminements des individus nous ont aussi préoccupés tout au cours de la démarche que nous avons privilégiée.

Les renseignements qui émanent de cette étude contribuent à l'avancement des connaissances puisqu'ils visent un domaine encore peu exploré, c'est-à-dire l'insertion professionnelle des individus qui ont complété une formation au Conservatoire de musique du Québec. Il ressort principalement de ces travaux que, parmi les individus dont les parents avaient des activités socioculturelles élevées et qui se retrouvent sans travail pendant la semaine contrôle, une quantité appréciable pourrait poursuivre des études dans une autre institution à la suite du CMQ. Le CMQ n'offre pas de niveau supérieur au quatrième cycle (cycle qui correspond à la maîtrise par rapport à l'ordre d'enseignement universitaire). Cette situation s'observe principalement chez les individus qui ont opté pour une discipline principale instrumentale associée aux cordes frottées. De leur côté, les individus qui pratiquent un instrument associé traditionnellement à l'orchestre de type symphonique ${ }^{21}$ et qui travaillaient pendant la même période se retrouvaient moins souvent à occuper des emplois à temps plein que les autres instrumentistes. Nous pensons qu'encore une fois, cette situation peut trouver une explication - en partie du moins - dans le fait que ces individus poursuivent des études additionnelles après le CMQ.

Le domaine de la musique professionnelle semble aussi assez sexué. Les filles optent essentiellement pour les disciplines instrumentales et, si on examine la situation en France, on observe que les aspirations professionnelles des filles sont moins axées que celles des garçons vers la carrière de musicien professionnel. 
D'autre part, il existe une relation directe (négative) entre les activités socioculturelles des parents et l'âge du début des apprentissages des individus. De plus, l'âge du début des apprentissages musicaux des individus présente une corrélation avec la situation, mais surtout avec la correspondance entre la formation et l'emploi occupé pendant la semaine contrôle. C'est pourquoi nous pensons que les activités socioculturelles des parents pourraient avoir un lien - direct ou indirect avec la correspondance formation-emploi. Rappelons que la correspondance formation-emploi est un indicateur de l'insertion professionnelle dans cette étude.

Parmi les individus qui ont obtenu une récompense (ou note) faible à la dernière année achevée au CMQ et qui travaillaient pendant la semaine contrôle, une forte proportion de ces individus occupaient des emplois correspondant à leur formation. Cette situation laisse penser que les employeurs ne sont pas très influencés par cette caractéristique.

De plus, parmi les individus qui pratiquent des instruments associés à l'orchestre de type symphonique et qui travaillaient pendant la semaine contrôle, on constate que le statut socio-économique du père semble influencer le type d'emploi que ces individus occupent. En effet, les individus qui travaillent dans des domaines correspondant à leur formation proviennent massivement des milieux populaires tandis que ceux qui travaillent dans des domaines non correspondant proviennent principalement de milieux aisés.

Malgré les visées d'insertion professionnelle de l'Institution, on peut penser que les finissantes et les finissants du CMQ qui travaillent dans leur domaine occupent principalement des emplois associés au style populaire car l'accès y est moins difficile que celui des orchestres classiques d'une part, mais aussi parce que ces emplois correspondent davantage aux statuts socioculturel et socio-économique d'origine. De plus, la musique populaire se prête à la pratique de presque toutes les familles instrumentales, ce qui n'est pas le cas du répertoire des orchestres de type symphonique.

Enfin, les musiciennes et les musiciens font face à des débouchés (reliés à leur domaine) souvent précaires et peu nombreux. Ainsi, le concept de stabilité tel que défini par Dupaquier (1986) est une 
dimension qui ne correspond pas de façon spécifique à la réalité des finissantes et des finissants du CMQ sur le plan professionnel. La stabilité est une dimension de l'insertion professionnelle qui suppose un travail à temps plein, sur une base régulière. La correspondance entre l'emploi et la formation semble mieux adaptée pour vérifier l'insertion professionnelle de cette clientèle.

\section{Références}

Archambault, É., et al. (1990). L'insertion professionnelle des élèves issus des écoles d'arts plastiques. Sous la direction de J. Cardona, Paris, ministère de la Culture et de la Communication.

Blishen, R.B., Carroll, W.K., \& Moore, C. (1987). The 1981 socio-economic Index for Occupation in Canada. Revue canadienne de sociologie et d'anthropologie, 24(4), 465-488.

Blume, H. (1978). Une école nationale de musique pour le Canada. Ottawa, ON: Conseil des arts du Canada.

Bourdieu, P. (1979). Les trois états du capital culturel. Actes de la recherche en sciences sociales ( 30 novembre).

Carrier, G., Cloutier, R., Laforce, L., Perron, M., \& Trottier, C. (1991). Relance Laval: Formation universitaire et insertion professionnelle des titulaires d'un grade de l'Université Laval; promotion de 1988, premier cycle. Sainte-Foy, PQ: Faculté des sciences de l'éducation, Laboratoire de recherches en administration et politique scolaires.

Duchesnay, A.J. (1980). Un regard sur le chemin. Québec: Conservatoire de musique et d'art dramatique du Québec, ministère des Affaires culturelles. Texte ronéotypé.

Dupaquier, M., Fourcade, B., Gadrey, N., Parel, J-J., \& Rose, J. (1986). L'insertion professionnelle. In L. Tanguy (éd), L'introuvable relation formation/emploi. Un état de la recherche en France, (pp. 35-88). Paris: La Documentation Française.

Gurvitch, G. (1963). La vocation actuelle de la sociologie. Paris: Presses Universitaires de France, 3e édition, vol. 1, pages 11 à 14.

Hennion, A., Martinat, F., \& Vignolle, J.-P. (1983). Les conservatoires et leurs élèves: Rapport sur les élèves et anciens élèves des écoles de musique agréées par l'État. Paris: La Documentation Française. 
Lamoure, J., \& Viney, X. (1982). L'entrée dans la vie active des jeunes sortant d'IUT et de STS: complémentarités et concurrences. Paris: La Documentation Française.

Painchaud, C. (1992). Statistiques, clientèle étudiante, 30 septembre 1992. Québec: Direction générale des conservatoires de musique du Québec.

Perron, D. (1995). Les aspirations professionnelles et l'insertion professionnelle des individus qui ont complété une formation au Conservatoire de musique $d u$ Québec. Mémoire de maîtrise en administration et politique scolaires, Faculté des sciences de l'éducation, Université Laval, Sainte-Foy, PQ. Québec.

Robert, M. (1988). Fondements et étapes de la recherche scientifique en psychologie. Paris: Edissem.

Rocher, G. (1969). Introduction à la sociologie générale: l'action sociale. Montréal, PQ: HMH.

Rousseau, C. (1986). Vivre de la musique: Conseils pratiques à l'intention des professionnels de la musique. Québec, PQ: Les Publications du Québec.

Tanguy, L. (éd). (1986). L'introuvable relation formation/emploi. Un état de la recherche en France. Paris: La Documentation Française.

Thibault, P. (1991). Le régime pédagogique et les programmes d'études en vigueur dans les conservatoires de musique. Québec, PQ: Ministère des affaires culturelles, direction générale des conservatoires.

Trottier, C., Diambomba, M., \& Perron, M. (1994). Les cheminements scolaires et l'insertion professionnelle des étudiants de l'université: perspectives théoriques et méthodologiques. Sainte-Foy, PQ: Presses de l'Université Laval.

Vincens, J. (1989). Réflexion sur l'insertion dans la vie active. Toulouse: Centre d'études juridiques et économiques de l'emploi. Université des sciences sociales.

\section{Notes}

1 L'Institution québécoise - dans son ensemble - comprend deux divisions: 1) le Conservatoire de musique; 2) le Conservatoire d'art dramatique. Toutefois, cet article porte que sur les finissantes et finissants en musique. C'est pourquoi nous parlerons le plus souvent du Conservatoire de musique du Québec (CMQ) exclusivement. 
2 «Sociétés globales et groupements particuliers se constituent à partir de différents types de liaisons sociales. C'est ce que souligne Gurvitch: on ne saurait étudier avec quelque précision un groupement concret quel qu'il soit, sans d'une part l'intégrer dans une société globale particulière, sans d'autre part décrire la constellation singulière du microcosme de liaisons sociales qui le caractérise » (Rocher, 1969: 9).

3 Dans ce cas, la filière de formation a des particularités puisque la fréquentation du Conservatoire de musique - et la possibilité d'obtenir une chaise à l'orchestre par la suite - constituent pour les individus issus des classes sociales défavorisées et intermédiaires un moyen d'ascension sociale. Il en est tout autrement pour les individus provenant de milieux socioculturels et socioéconomiques favorisés pour qui la musique représente un ajout au curriculum et à la culture générale, mais moins souvent une visée professionnelle.

4 Au CMQ le terme «élève» s'applique à tous les individus en situation de formation.

5 Pour plus de détails quant à la liste des disciplines principales offertes, voir Thibault 1991, p. 36.

6 Un questionnaire a été rejeté parce que mal rempli.

7 Étude qui porte sur la formation universitaire et l'insertion professionnelle des titulaires d'un grade de premier cycle de l'Université Laval, toutes disciplines confondues.

8 Il faut insister sur le fait que le CMQ vise à former des musiciennes et des musiciens professionnels, pas des enseignantes ou des enseignants, ni des mélomanes, etc.

${ }^{9}$ Précisons toutefois qu'il existe une relation significative entre les trois indicateurs qui définissent - dans notre étude - l'insertion professionnelle, c'est-à-dire la situation d'emploi, le statut d'emploi et la relation formation/ emploi. Pour plus de détails, voir Perron, 1995, pp. 133-134.

10 Semaine du 14 novembre 1994.

11 La littérature est abondante à cet égard, nous pensons à Boudon, R., Bourdieu, P., Passeron, J.-C., etc.

12 Nous avons demandé aux individus dans quelle mesure les parents ou tuteurs: 1) assistaient à des spectacles musicaux; 2) fréquentaient les musées; 3) allaient au théâtre; 4) écoutaient de la musique; 5) allaient au cinéma; 6) allaient à des spectacles de danse. 
13 Les trois catégories obtenues par Relance Laval (Carrier et al., 1991)et formées à partir de l'occupation du père - correspondent exactement aux mêmes points de coupe que ceux auxquels nous sommes arrivés dans ce travail, bien que les populations ne fréquentaient pas les mêmes institutions.

14 Notons que les décimales n'existent pas dans les notes décernées au CMQ.

15 Voir tableau $n^{\circ} 4$.

16 Voir tableau n ${ }^{\circ} 5$.

17 Le Conservatoire de musique du Québec a comme objectif de former des musiciennes et des musiciens professionnels essentiellement. L'enseignement ne fait pas partie des visées de cette Institution.

$18 \mathrm{Au} \mathrm{CMQ}$, les disciplines principales non instrumentales comprennent: l'analyse, la composition, le contrepoint, la direction d'orchestre, la fugue, l'harmonie et l'histoire de la musique.

19 Voir tableau n ${ }^{\circ} 6$.

20 Ces individus pourraient investir plus d'énergie à la quête d'un emploi dans leur domaine - que celles et ceux qui ont obtenu de meilleures performances à la dernière année au CMQ - étant donné qu'une formation additionnelle leur apparaitrait beaucoup moins attrayante.

D'autre part, la plupart des engagements dans les orchestres canadiens se font par auditions. Dans son rapport, Helmut Blume (1978) indique toutefois qu'un grand nombre d'étudiants doués sont mal préparés à affronter la réalité de l'orchestre. « Les musiciens étrangers sont plus positifs, plus entreprenants et plus disposés à se déplacer. Et, ce qui est triste, ils sont d'habitude meilleurs. Très peu de Canadiens demandent à passer des auditions (p.5) ». La coutume veut aussi que ces auditions soient faites souvent « derrière rideau » de façon à ne pas influencer les membres du jury. On peut penser que la récompense (ou note) décernée est conforme à la qualité des musiciennes et des musiciens, mais que celle-ci n'est pas un critère de sélection.

21 Incluant aussi les cordes frottées qui constituent la majeure partie de l'effectif des orchestres de type symphonique. 